Technische

Universität

Berlin

\author{
Xu Wang, Xiaotong Sun, Huadong Yu, Jinkai Xu, Zhanjiang Yu, \\ Yiquan Li, Valentin L. Popov
}

\title{
Research on the micro-hole texture forming of PCD tool surface
}

Open Access via institutional repository of Technische Universität Berlin

\section{Document type \\ Conference paper | Accepted version}

(i. e. final author-created version that incorporates referee comments and is the version accepted for publication; also known as: Author's Accepted Manuscript (AAM), Final Draft, Postprint)

This version is available at

https://doi.org/10.14279/depositonce-15074

\section{Citation details}

X. Wang et al. (2021) Research on the micro-hole texture forming of PCD tool surface. 2021 IEEE International Conference on Manipulation, Manufacturing and Measurement on the Nanoscale (3M-NANO), 2021, pp. 365-368, https://doi.org/10.1109/3M-NANO49087.2021.9599816.

(C) 2021 IEEE. Personal use of this material is permitted. Permission from IEEE must be obtained for all other uses, in any current or future media, including reprinting/republishing this material for advertising or promotional purposes, creating new collective works, for resale or redistribution to servers or lists, or reuse of any copyrighted component of this work in other works.

\section{Terms of use}

This work is protected by copyright and/or related rights. You are free to use this work in any way permitted by the copyright and related rights legislation that applies to your usage. For other uses, you must obtain permission from the rights-holder(s). 


\title{
Research on the micro-hole texture forming of PCD tool surface
}

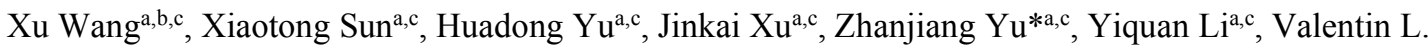 \\ Popov*b, \\ a. National and Local Joint Engineering Laboratory for Precision Manufacturing and Detection \\ Technology, Changchun University of Science and Technology, Changchun 130012, Jilin, PR China; \\ b. Technische Universität Berlin, 10623, Berlin, Germany, \\ c. National and Local Joint Engineering Laboratory for Precision Manufacturing and Detection \\ Technology \\ Changchun 130012, Jilin, PR China \\ Technische Universität Berlin, 10623, Berlin, Germany \\ v.popov@tu-berlin.de \\ 13514310244@163.com
}

\begin{abstract}
Based on the research on the forming mechanism of textured PCD tool surface, the nanosecond laser is used to study the influence of laser machining parameters on the size and topography of PCD tool surface micro texture. The micro-hole texture is prepared on the surface of the PCD tool, and a single factor experiment is designed to study the influence of laser power, pulse frequency and defocusing amount on the micro-hole texture. The results show that, the micro-hole diameter increases gradually with the laser power, but decreases with the pulse frequency; the overall micro-hole diameter tends to increase with the defocus. The pulse frequency has the greatest impact on the micro-hole diameter, followed by the defocus amount, and finally the laser power. The influence of different parameters on the surface recast layer is also completely different. As a result, the surface and laser power are the main factors that affect the surface recast layer.
\end{abstract}

Keywords-PCD; Forming mechanism; Micro-hole diameter.

\section{INTRODUCTION}

Polycrystalline diamond tools have the characteristics of high cutting efficiency, low friction coefficient, low thermal expansion coefficient, good thermal conductivity, low affinity with materials and high machining accuracy, so it is widely used in automobiles, aerospace and micro-components. People use high-pressure synthesis technology to synthesize polycrystalline diamond (PCD), which solves the problem of scarcity and high price of natural diamond [1]. Nevertheless, the use of PCD tools to machine some super hard materials or metal matrix composites will still have edge chipping, and the machining of some metal materials with higher plasticity will also cause sticking. Micro-texture improves the friction behavior of the surface by reducing the contact area, thereby improving the wear resistance of the tool. At present, there are few researches on the surface texture of PCD tools, so it is very important to study the preparation of PCD tools surface texture to improve the cutting performance and life of the tools. The preparation of hole arrays on the tool surface is the most economical and efficient way to solve this problem [2-5].

At present, the preparation of the surface texture of PCD tools mainly includes laser machining and electrical discharge machining (EDM). EDM machining is pretty difficult for 
preparation of smaller holes, and the machining cycle is longer. Wang et al. [6] used EDM to machine micro-holes in PCD materials. The minimum size of the micro-holes is $130 \mu \mathrm{m}$ and the maximum is $200 \mu \mathrm{m}$. Su et al. [7] used a fiber laser to prepare micro-grooves on the surface of the PCD tool, and the average width of the microgrooves was between 56-60 $\mu \mathrm{m}$. And the results show that $\mathrm{PCD}$ textured tool has improved the cutting performance. Knowles [7] used three high-intensity lasers (with magnitude of order $\mathrm{GW} / \mathrm{cm}^{2}$ ) to prepare textures for metals, ceramics, and polymers. The smallest hole size can reach $50 \mu \mathrm{m}$. Khan and Zheng et al. [8-9] used nanosecond lasers to prepare textures of nickel-coated tungsten carbide and cemented carbide materials. The size of the hole texture was about $50 \mu \mathrm{m}$, and the size of the groove-texture was about 50-160 $\mu \mathrm{m}$. They all reduced the wear of the tool and the adhesion of the workpiece surface, and improved the surface quality of the workpiece. Uhlmann [10] studied the preparation and cutting performance of CVD diamond texture tools. The texture with a size of $60 \mu \mathrm{m}$ was prepared on the surface of CVD diamond tools. The results show that the texture can effectively reduce the cutting force. From the point of view of both machining method and machining efficiency, the better choice for micro-hole machining on the surface of PCD tools is laser machining.

There have been many researches on the machining of texture on the surface of PCD tools by laser, but quite few on the influence of laser machine parameters on the microscopic morphology and micro-hole diameter of the surface hole array of PCD tools. In this work a single-factor experiment is designed for study on the preparation of PCD surface micro-holes. The effects of laser power, pulse frequency and defocusing amount on the surface micro-morphology and micro-hole diameter will be studied. A quantitative relationship will be obtained to analyze the degree of the influence.
The micro-hole array textured PCD tool is prepared by selecting appropriate machine parameters, which provides a basis for the selection of the machine parameters of the micro-hole array textured PCD tool.

\section{EXPERIMENTAL EQUIPMENT AND METHODS}

The PCD tool produced by Xiamen Golden Egret Special Alloy Co., Ltd. was selected in the experiment. Its material properties are shown in Table I. The laser parameters for the single factor experiment are chosen as shown in Table II. The effects of 4 different laser powers, seven defocusing amounts varying from $-3 \mu \mathrm{m}$ to $3 \mu \mathrm{m}$, and 9 pulse frequencies ranging from $20 \mathrm{kHz}$ to $100 \mathrm{kHz}$ on the surface texture morphology and hole diameter of PCD tools are respectively investigated. The laser is a nanosecond laser with a maximum power of $20 \mathrm{~W}$, and the incident angle is $90^{\circ}$, as shown in Fig 1. Due to the high hardness of PCD tools, the high power is selected from $70 \%$ of the maximum power to the full power. Three holes are prepared for each combination of the three laser parameters, and the value of hole diameter is averaged for analysis. The observation and detection are carried out on a scanning electron microscope (SEM).

\section{TABLE I. PCD TOOL PHYSICAL PARAMETERS}

\begin{tabular}{|l|c|}
\hline Density $\left(\mathbf{g} / \mathbf{c m}^{\mathbf{3}}\right)$ & $3.47-4.12$ \\
\hline Elastic Modulus (GPa) & $820-1050$ \\
\hline Hardness & HV8000-10000 \\
\hline Thermal Conductivity $(\mathbf{W} /(\mathbf{m} \cdot \mathbf{K}))$ & $500-2000$ \\
\hline Thermal Expansion Coefficient & $0.9-4.8$ \\
\hline
\end{tabular}

TABLE II. SINGLE FACTOR MACHINING PARAMETER

\begin{tabular}{|l|c|c|}
\hline $\begin{array}{l}\text { Power } \\
(\mathbf{W})\end{array}$ & $\begin{array}{c}\text { Defocus Amount } \\
(\boldsymbol{\mu m})\end{array}$ & $\begin{array}{c}\text { Pulse Frequency } \\
(\mathbf{k H z})\end{array}$ \\
\hline $70 \%$ & -3 & 20 \\
\hline $80 \%$ & -2 & 30 \\
\hline $90 \%$ & -1 & 40 \\
\hline $100 \%$ & 0 & 50 \\
\hline
\end{tabular}




\begin{tabular}{|l|l|l|}
\hline & 1 & 60 \\
\hline & 2 & 70 \\
\hline & 3 & 80 \\
\hline & & 90 \\
\hline & & 100 \\
\hline
\end{tabular}

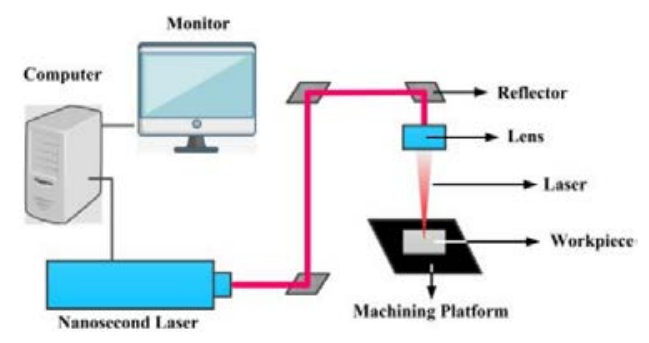

Fig. 1 Schematic diagram of nanosecond laser machining system

The PCD tool is cleaned before and after machining by ultrasonic cleaning machine. Fig. 2 (a) is a side cross-sectional view of the PCD tool after machining. The laser enters into the PCD tool through periodic pulses, and high-energy makes the material melt and evaporate quickly, finally produces micro-hole. As shown in the schematic diagram of Fig.2(c), the PCD material absorbs the energy in the laser irradiated area, and the energy is converted into heat, which accumulates in a very short time, so that the material rapidly oxidizes, changes phase, and begins to melt. As the temperature continues to increase, the PCD material is evaporated and sprayed to remove the material, and a recast layer is possibly formed on the surface of the tool, as shown in Fig. 2(b).
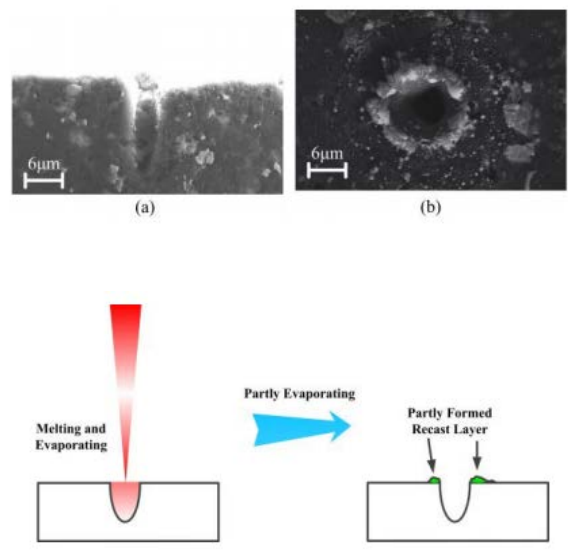

Fig. 2 SEM image and schematic diagram of laser machining PCD tool (a) Side view of laser machining PCD tool, (b) Top view of laser machining PCD tool. (c) Schematic diagram of micro hole producing.

\section{EXPERIMENTAL RESULTS AND ANALYSYS}

As shown in Fig. 3, as the pulse frequency increases from $20 \mathrm{kHz}$ to $100 \mathrm{kHz}$, the micro-hole diameter decreases from about $5 \mu \mathrm{m}$ to about 2 $\mu \mathrm{m}$. The main reason is that as the pulse frequency increases, the working time between a single pulse and the material decreases, so that the energy density per unit area of the machined material becomes lower, which leads to a decreasing of micro-hole diameter.

As the laser power increases from $14 \mathrm{~W}$ to 20 $\mathrm{W}$, the acting area of laser on the PCD tool material becomes larger, then the diameter of the micro-hole increases. Due to the Gaussian distribution of laser energy density, the laser spot diameter limits the diameter of the micro-hole, so that the hole size increases as the power increases. For a certain value of pulse frequency and defocusing amount, the maximum difference of hole diameter is about $0.962 \mu \mathrm{m}$, and the minimum is about $0.464 \mu \mathrm{m}$. It can be seen that the influence of the laser power in this range on the hole size is not obvious than that of the pulse frequency.

Considering the effect of the defocusing amount, it is found that the hole's diameter fluctuates by $0.192 \sim 1.295 \mu \mathrm{m}$. For the power $70 \%$, the largest diameter of the micro-hole is found at the defocus -3 . For the power $80 \%$ and $90 \%$, the change of the hole diameter is not obvious when the defocus amount increases. For the power is $100 \%$, the largest hole is found at the defocus 3 and -3 . As the amount of defocus increases in both directions, the overall hole's diameter shows an increasing trend. 


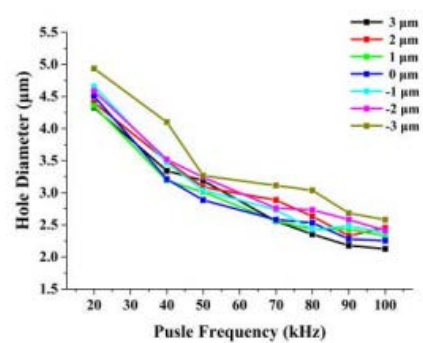

(a)

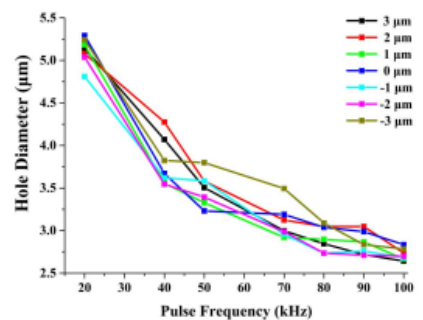

(b)

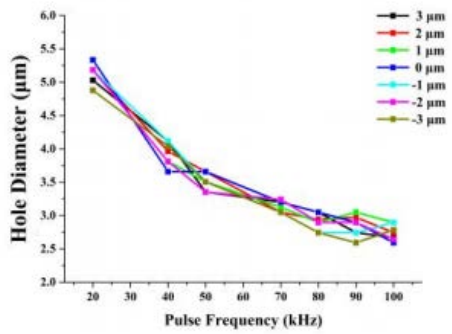

(c)

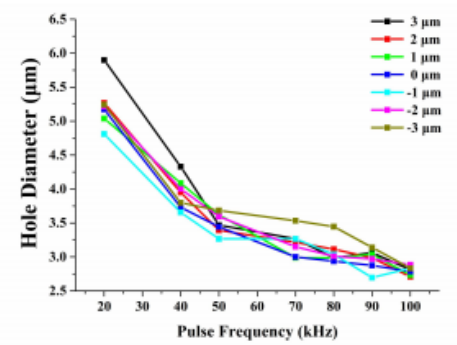

(d)

Fig. 3 The effect of pulse frequency and defocusing amount on hole diameter under different laser powers. The dependence of pulse frequencies on the hole diameter for different values of defocus with (a) $70 \%$ power, (b) $80 \%$ power, (c) $90 \%$ power and (d) $100 \%$ power.
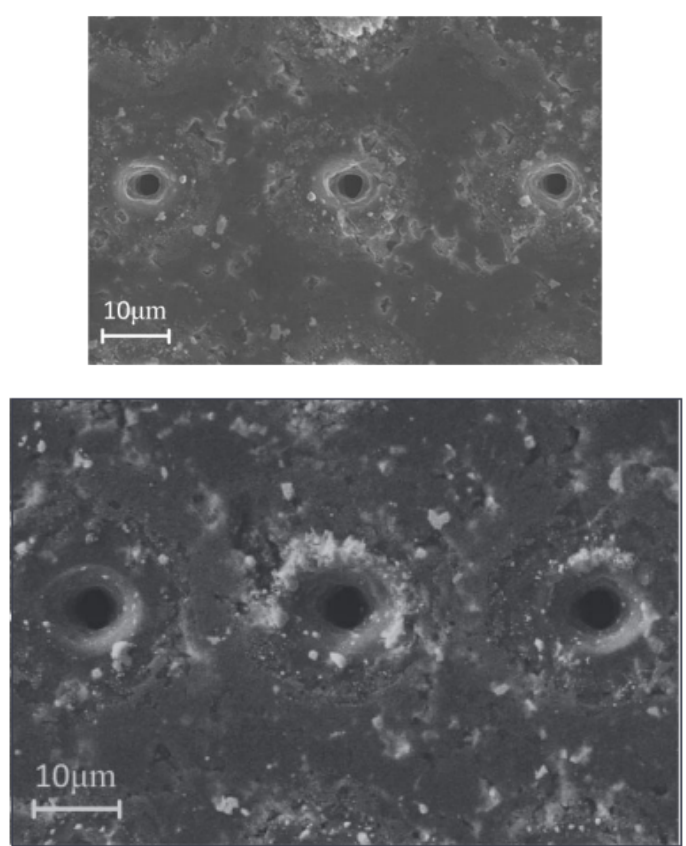

Fig. 4 The micro-hole surface micro-morphology under different machining parameters.

A few examples of the PCD surface micromorphology of the machined hole are shown in Fig 4. There are mainly three types: (1) the surface is flat and there is almost no recast layer; (2) the surface is bowl-shaped and there is almost no the recast layer; (3) the surface is bowl-shaped and recast layers exist. The holes without bowl-like structure were prepared when the focus was located on the surface or below the surface of the workpiece (negative defocusing). With a gradually increasing the laser energy, a flat micro-hole structure on the tool surface is produced. For the bowl-shaped structure, since the laser focus is above the surface of the workpiece, the energy decreases from the focus to the surface of the workpiece, and then the bowl-shaped structure is produced. The surface recast layer is mainly determined by the laser power. In the case of focusing or positive defocusing, high power will cause the surface to produce an excessively large recast layer area.

To predict the cutting performance, we numerically simulated the cutting of this textured tool with $\mathrm{SiCp} / \mathrm{Al}$ composite. The cutting model was established by ABAQUS software. The 
maximum stress at the tool texture was compared for different hole diameters and the holes with and without rounded corners. The stress distribution on the surface is shown in Fig. 5. It can be seen that with an increasing hole diameter (from left to right), the maximum stress of the tool's texture becomes gradually larger. And it is smaller when the texture has rounded corners (in the bottom row). Therefore, the texture with rounded corners can reduce the maximum stress at the tool's texture during the machining, thereby reducing tool wear.
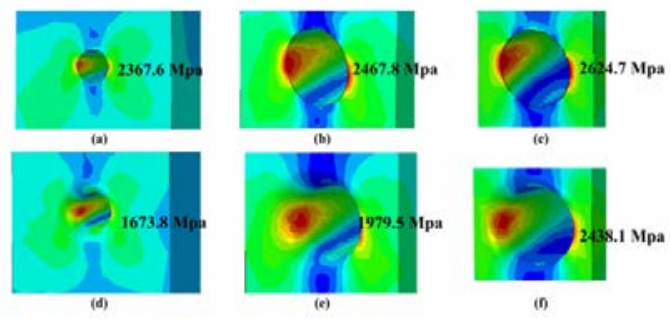

Fig. 5 The maximum stress in the machining of textured PCD tools on $\mathrm{SiCp} / \mathrm{Al}$ composite. The texture of hole diameter of (a) $2 \mu \mathrm{m}$, (b) $4 \mu \mathrm{m}$ and (c) $6 \mu \mathrm{m}$ without rounded corners, and the same hole sizes $(d)(e)(f)$ with rounded corners.

\section{CONCLUSIONS}

The effects of laser power, pulse frequency and defocusing amount on the micro-hole diameter and micro morphology of the PCD tool surface was studied, and the conclusions are as follows:

(1) By changing the laser power, pulse frequency and defocus amount, the size and micro-morphology of the surface texture of the PCD tool can be controlled;

(2) The diameter of the micro-holes on the surface of the PCD tool decreases significantly with the pulse frequency; shows a decreasing With the increase of the laser power and the defocusing amount, the hole diameter shows an increasing trend. The order of the influence of laser parameters on the diameter of the micro-hole is: pulse frequency $>$ defocus amount $>$ laser power.

(3) The micro-hole machined by laser on the surface of PCD tools have mainly three forms: relatively flat surface without recast layer in the micro-hole; bowl-shaped surface without recast layer; bowl-shaped surface with heavy cast layer. The bowl-shaped structure is determined by the defocus amount, and the recast layer is mainly determined by the laser power.

(4) The texture with rounded corners can reduce the stress concentration, extend the effectiveness of the textured tool, and further improve the tool life.

\section{ACKNOWLEDGMENT}

This work was supported by the National Key Research and Development Plan Project (No.2018YFB1107400), the National Key Research and Development Plan Project (No.2018YFB1107403), the "111" Project of China (No.D17017), Jilin Province Scientific and Technological Development Program (No.20190101005JH, No.20180201057GX, No.20190302076GX), and Science Fund for Youth Scholar of Changchun University of Science Technology (No.XQNJJ-2018-09).

\section{REFERENCES}

[1] R. Lindvall, F. Lenrick, H. Persson, R. M'Saoubi, J.-E. Ståhl, V. Bushlya. "Performance and wear mechanisms of $\mathrm{PCD}$ and pcBN cutting tools during machining titanium alloy Ti6A14V," Wear,2020.

[2] H. M. Pen, X. Liu, N. Ni, Z. T. Chen, F. Liu, C. X. Yue. "Experimental Research on PCD Tool Turning SiCp/Al Composites," Aeronautical Manufacturing Technology, vol. 11, pp. 14-19, 2020.

[3] D. Arulkirubakaran, \& V. Senthilkumar, "Performance of TIN and TIAIN coated micro-grooved tools during machining of Ti-6AL-4V alloy," International Journal of Refractory Metals \& Hard Materials, vol. 62, pp. 47-57, 2015.

[4] R. B. Soares, A. M. P. Jesus, R. J. L. Neto, B. Chirita, P. A. R. Rosa,A. Reis. "Comparison between cemented carbide and PCD tools onmachinability of a high silicon aluminum 
alloy," Journal of Materials Engineering and Performance,vol. 26, pp. 4638-4657, 2017.

[5] B. Dan, G. Anthony, "Homogeneous and structured PCD/WC-Co materials for drilling" Diamond \& Related Materials, vol. 10, 2004.

[6] D. Wang, W. S. Zhao, L. Gu, X. M. Kang, "A study on micro-hole machining of polycrystalline diamond by micro-electrical discharge machining," Journal of Materials Processing Tech., vol. 12010.

[7] Y. S. Su, Z. Li, L. Li, J. B. Wang, H. Gao,G. Wang, "Cutting performance of micro-textured polycrystalline diamond tool in dry cutting," Journal of Manufacturing
Processes, pp. 1-7, 2017.

[8] M. A. Khan, K Gupta, "A study on machinability of nickel based superalloy using micro-textured tungsten carbide cutting tools" Materials Research Express, vol. 1, pp. $1-7,2020$

[9] K. Zheng, F. Yang, N. Zhang, Q. Y. Liu, F. L. Jiang, "Study on the Cutting Performance of Micro Textured Tools on Cutting Ti-6Al-4V Titanium Alloy," Micromachines, vol. 2,2020 .

[10] E. Uhlmann, D. Schröter, "Process behaviour of micro-textured CVD diamond thick film cutting tools during turning of Ti-6Al-4V," pp. 25-30, 2020. 